\title{
Relationship between the Emulsion Stability and Phospholipid Distribution in the Aqueous Phases Inside and Outside of an Emulsion Droplet
}

\author{
Kazuhiro CHIBA and Masahiro TADA \\ Tokyo University of Agriculture and Technology, \\ Laboratory of Bio-organic Chemistry, \\ Fuchu-shi, Tokyo 183, Japan
}

Received September 5, 1989

\begin{abstract}
The distribution of phospholipids between the inside and outside of an emulsion droplet was studied in relation to the emulsion stability. The emulsion was prepared with various mixtures of phosphatidylcholine (PC) and lysophosphatidylcholine (LPC), n-decane and water containing $\mathrm{HCl}$, $\mathrm{NaCl}$ or $\mathrm{NaOH} .{ }^{13} \mathrm{P}-\mathrm{NMR}$ spectra of the emulsion in the presence of lanthanide ion $\mathrm{Pr}^{3+}$ allowed quantitative measurement of the phospholipids in the internal and external aqueous phases, and in multilamellar vesicles. By a phospholipase $\mathrm{A}_{2}$ treatment of $\mathrm{PC}$ facing the continuous aqueous phase, most of the internal phospholipids were suggested to be composed of PC. The ratio of the phospholipids inside and outside changed with the emulsifying conditions, and was well correlated with the emulsion stability. Thus, it is suggested that the phospholipid distribution in the emulsion of PC and LPC showed the change of their hydrophile-lipophile balance and of the interfacial absorption force.
\end{abstract}

The composition of phospholipids varies among species, organs, and even between the inside and outside of biomembranes. ${ }^{1)}$ The asymmetrical distribution of membrane phospholipids allows a structural barrier to be formed such as cell membranes or liposomes. ${ }^{2-6)}$ A structural barrier like the wateroil-water type of emulsion has often been observed by emulsifying two or more kinds of emulsifiers of different hydrophile-lipophile balance. $^{7,8)}$ Phosphatidylcholine (PC) is one of the most common phospholipids for constructing biomembranes. Lysophosphatidylcholine (LPC), although it exists as minor component in phospholipids, has higher water solubility and emulsifying properties, and interacts with PC by the wedge mechanism to change the structure and the fluidity of a membrane..$^{9,10)}$ Thus, we have been studying the formation of emulsions with the diverse interaction of PC and $\mathrm{LPC}$.

Previously, we investigated the headgroup motion of emulsified PC and LPC by the line widths of ${ }^{31} \mathrm{P}-\mathrm{NMR}$ and the $T_{2}{ }^{*}$ relaxation time of ${ }^{13} \mathrm{C}-\mathrm{NMR}$ spectra, and found that the emulsion stability was well correlated with the headgroup motional properties. ${ }^{11,12)}$ Namely, in the emulsion system composed of PC, water and $n$-decane, the line width of the phosphorus signal was extremely broad, indicating that most of the PC had aggregated to form lamella. On the other hand, an equimolar mixture of PC and LPC gave a stabler emulsion than that of PC alone, and the phosporus signal narrowed. This result suggested that the PC was locate at the interface of the emulsion by fusion of the lamella structure with LPC; consequently, the headgroup of PC had motional freedom. Moreover, as PC and LPC change their hydrophile-lipophile balance depending on the aqueous phase conditions, it can be expected that some of the emulsifying conditions allow the formation of an internal aqueous phase, and phospholipids would be distributed partly at the internal interface of the oil particles.

In this paper, we discuss the relationship between the formation of the internal aqueous phase and the stability of an emulsion containing PC and LPC. In order to study the distri- 
bution of phospholipids on the inside, outside, at the interfaces and in the lamella. ${ }^{31} \mathrm{P}-\mathrm{NMR}$ was used in the presence of the lanthanide probe ion $\mathrm{Pr}^{3+}$ in the continuous aqueous phase of the emulsion.

\section{Materials and Methods}

Preparation of aqueous dispersions and emusions of phospholipids. PC and LPC were obtained from egg lecithin as previously described, ${ }^{11}$ and were separately dispersed in deionized water prior to mixing. After they had been mixed and the aqueous phases conditioned by the addition of a $0.1 \mathrm{M} \mathrm{HCl}, \mathrm{NaCl}$ or $\mathrm{NaOH}$ solution, they were agitated by a disperser (Physcotron fitted with an NS-10 generator shaft) at $20,000 \mathrm{rpm}$. These aqueous dispersions of the mixture of PC and LPC were submitted to ${ }^{31} \mathrm{P}$ NMR measurements in the absence and presence of $5 \mathrm{mM}$ $\mathrm{PrCl}_{3}$. The emulsion was obtained by the addition of $n$ decane $(5 \mathrm{ml})$ dropwise to the aqueous dispersion of phospholipids $(5 \mathrm{ml})$ with agitation by the disperser. The emulsion stability was evaluated by the change of particle size distribution, which was measured by a particle size counter (Coulter Counter, Model $\mathrm{T}_{\mathrm{AII}}$ ), and the ratio of the separated aqueous layer after $24 \mathrm{hr}$ standing at $25^{\circ} \mathrm{C}$. The emulsion was also analyzed with a ${ }^{31} \mathrm{P}-\mathrm{NMR}$ spectrometer in the presence and absence of $\operatorname{Pr}^{3+}$.

Measurements of the ${ }^{31} P-N M R$ spectra. ${ }^{31} \mathrm{P}-\mathrm{NMR}$ spectra were recorded on a JEOL GX-270, employing a $45^{\circ}$ pulse, with $32-\mathrm{k}$ data points, a $4000 \mathrm{~Hz}$ spectral window and $1.5 \mathrm{sec}$ pulse delay, the protons not being decoupled. The ionic concentration of $\mathrm{Pr}^{3+}(5 \mathrm{mM})$ was obtained by pipetting $10 \mu \mathrm{l}$ of a $\mathrm{D}_{2} \mathrm{O}$ stock solution of $\mathrm{PrCl}_{3}$ into $\mathrm{NMR}$ tubes. It has been shown that these peak areas can be used reliably for a quantitative analysis of phospholipids. ${ }^{13}$ ) The down-field shift is dependent on the concentration of $\mathrm{Pr}^{3+}$ because of the pseudo-contact shift effect of paramagnetic $\mathrm{Pr}^{3+}$ in rapid exchange between the phosphate sites on the headgroups. ${ }^{14)}$

Phospholipase $A_{2}$ treatment of the emulsified PC. The aqueous dispersion of $64 \mathrm{mM} P C$ and of equimolar mixtures of PC and LPC in various concentrations was. emulsified with $n$-decane and deionized water, and then treated with phospholipase $A_{2}$. An aqueous solution of phosphlipase $\mathrm{A}_{2}$ and $\mathrm{CaCl}_{2}$ was pipetted into the emulsion to give 200 units $/ \mathrm{ml}$ and $2 \mathrm{mM} \mathrm{Ca}^{2+}$ in the aqueous phase, and this was allowed to stand at $25^{\circ} \mathrm{C}$. The amount of unaltered PC was determined by HPLC ${ }^{11)}$ after the emulsion had been acidified with $0.1 \mathrm{M}-\mathrm{HCl}$ and dispersed in acetonitrile. In addition, dioctanoyl-PC was prepared by the acylation of glycerophosphorylcholine, ${ }^{15)}$ and the emulsion of dioctanoyl-PC (64 mM) was also treated by phospholipase $\mathrm{A}_{2}$ for use as a model emulsion in which most of the phosphatidylcholine faced a continuous aqueous phase.

\section{Results and Discussion}

${ }^{31} P$-NMR spectra of the aqueous dispersion and the emulsion of phospholipids in the absence and presence of $\mathrm{Pr}^{3+}$

The headgroup of LPC has motional freedom in the globular micelle to give a narrow ${ }^{31} \mathrm{P}$-NMR spectrum of $c .50 \mathrm{~Hz}$. The signal was shifted down-field with the addition of $\mathrm{Pr}^{3+}$, indicating that most of the polar headgroups of LPC faced a continuous aqueous phase. The phosphorus signal of $\mathrm{PC}$ in the aqueous dispersion was broadened to more than $2000 \mathrm{~Hz}$, which was caused by tight restriction of the headgroup in the lamella, and the chemical shift of the signal scarcely changed even in the presence of $\mathrm{Pr}^{3+}$. In the mixtures of $\mathrm{PC}$ and LPC, the phosphorus signals became broader with the increase of $\mathrm{PC} / \mathrm{LPC}$ ratio. When the ratio was more than $1 / 2,{ }^{31} \mathrm{P}-\mathrm{NMR}$ spectrum in the presence of $\mathrm{Pr}^{3+}$ clearly showed the existence of phospholipids in the lamella state.

Figure 1 shows ${ }^{31} \mathrm{P}-\mathrm{NMR}$ spectra of equimolar mixtures of PC $(32 \mathrm{~mm})$ and LPC ( $32 \mathrm{~mm}$ ) emulsified with $n$-decane and an aqueous phase of $10 \mathrm{~mm} \mathrm{HCl}, \mathrm{NaCl}$ or $\mathrm{NaOH}$. With $\mathrm{HCl}$ and $\mathrm{NaCl}$, no lamella signal was observed, and the signal was split by the addition of $\mathrm{Pr}^{3+}$, however, the phosphorus signal showed a complete down-field shift by the addition of $\mathrm{Pr}^{3+}$ before the emulsification. Thus, the split signal indicates the existence of discontinuous aqueous phases by forming a $\mathrm{W} / \mathrm{O} / \mathrm{W}$ emulsion, and a part of the phospholipids is presumed to face the internal aqueous phase, in which $\mathrm{Pr}^{3+}$ was not distributed by adding it into the continuous aqueous phase after emulsification. In the aqueous dispersion of PC and LPC, the amount of internal phospholipids was less than $10 \%$; however, it was markedly increased with emulsification. The line width of the inside signal was about $150 \mathrm{~Hz}$, and no broadening or down-field shift was observed despite the increase of $\mathrm{Pr}^{3+}$ in the continuous aqueous phase. The decrease of 

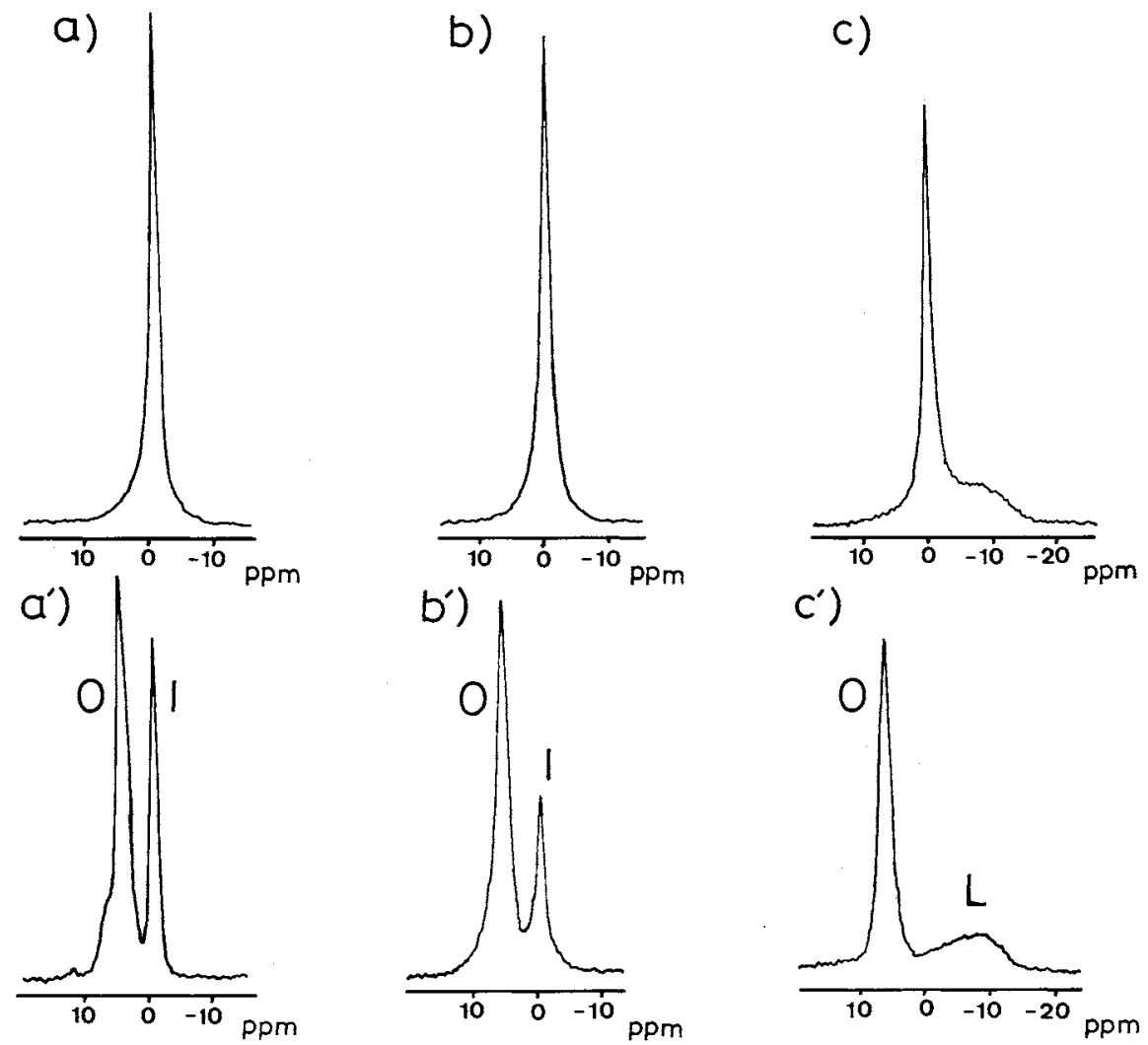

Fig. 1. ${ }^{31} \mathrm{P}-\mathrm{NMR}$ Spectra of Equimolar Mixtures of Emulsified PC and LPC in the Absence (a-c) Presence $\left(\mathrm{a}^{\prime}-\mathrm{c}^{\prime}\right)$ of $5 \mathrm{~mm} \mathrm{Pr}^{3+}$ in the Aqueous Phase.

The emulsion was composed of PC ( $32 \mathrm{~mm})$, LPC (32 mM), $n$-decane and water containing a, a') $10 \mathrm{~mm} \mathrm{HCl}, \mathrm{b}$, b') $\left.10 \mathrm{~mm} \mathrm{NaCl}, \mathrm{c}, \mathrm{c}^{\prime}\right) 10 \mathrm{~mm} \mathrm{NaOH}$. The spectra show the phospholipid signal inside (I), outside (O) and in the lamellar state $(\mathrm{L})$.

the phosphorus signals of lamella and the increase of the phospholipids distributed in the internal aqueous phase suggested that $\mathrm{PC}$ in multilamellar vesicles in the continuous aqueous phase had been promoted to pass into the $n$-decane phase, and that this PC had headgroup motional freedom on the internal interface. The ratio of internal/external phospholipids in the emulsion varied with the acid concentration, and reached a maximum in the conditions of $5 \mathrm{~mm}$ to $50 \mathrm{mM} \mathrm{HCl}$.

\section{Conversion of $P C$ and dioctanoyl-PC by phos-} pholipase $A_{2}$

The emulsions with PC, dioctanoyl-PC and the mixture of $\mathrm{PC}$ and $\mathrm{LPC}$ were treated with phospholipase $\mathrm{A}_{2}$, and the amounts of un- altered PC and dioctanoyl-PC according to time are shown in Fig. 2. The whole phosphorus signal of the dioctanoyl-PC in the emulsion showed a down-field shift; hence, their headgroups were confirmed to face the continuous aqueous phase, and they were hydrolysed to LPC (1-mono-octanoyl) rapidly by phospholipase $\mathrm{A}_{2}$. On the other hand, the conversion of $\mathrm{PC}$ in the emulsion was slow in comparison with that of dioctanoyl-PC. In the emulsion composed of PC, $n$-decane and water, the phosphorus signal shows that most of PC was in the lamellar state ${ }^{12}$; accordingly, it can be presumed that most of the $P C$ remained in multilamellar vesicles in the aqueous phase of the emulsion, and that it was prevented from being converted by phospholipase $A_{2}{ }^{16)}$ 
The emulsion of the equimolar mixtures of PC and LPC soon reached a plateau and the amount of unaltered PC gradually decreased. The result indicates that phospholipase $A_{2}$

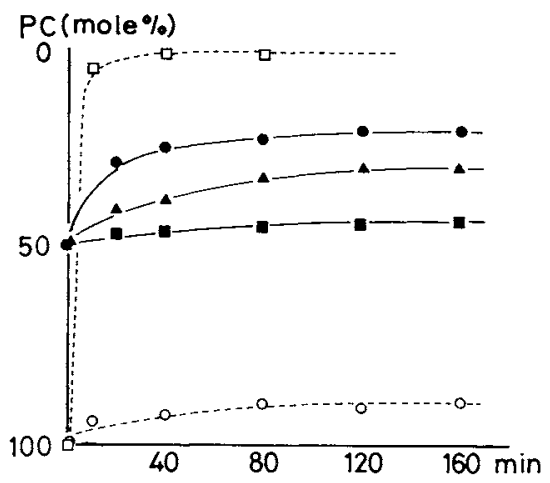

Fig. 2. Amounts of Unaltered PC by the Phospholipase $\mathrm{A}_{2}$ Treatment (mol\% of the Total Phospholipids) in the Emulsion Composed of Equivolumes of $n$-Decane and Deionized Water Containing $32 \mathrm{~mm} \mathrm{PC}$ and $32 \mathrm{~mm}$ $\mathrm{LPC}, \triangle 16 \mathrm{~mm} \mathrm{PC}$ and $16 \mathrm{~mm} \mathrm{LPC}, 8 \mathrm{~mm} \mathrm{PC}$ and $8 \mathrm{~mm}$ LPC, $\square 64 \mathrm{~mm}$ Dioctanoyl PC and $\bigcirc 64 \mathrm{~mm}$ PC.

The times on the abscissa indicate the periods of enzymatic treatment. quickly attacked $\mathrm{PC}$ facing the continuous aqueous phase, and after that, the reaction proceeded slowly with the permeation of phospholipase $\mathrm{A}_{2}$ or the exchange of phospholipids between the internal and external interfaces until all the PC had been converted to LPC. Accordingly, the amount of unaltered $\mathrm{PC}$ just after it reached a plateau can be regarded as the PC located inside the oil phase and in the multilamellar vesicles. The molar ratio of unaltered $\mathrm{PC}$ was increased by the dilution of the mixture of $\mathrm{PC}$ and $\mathrm{LPC}$ in the emulsion. In ${ }^{31} \mathrm{P}-\mathrm{NMR}$ spectra of their emulsified mixture, the ratio of phospholipids-located on the internal interface increased as the concentration of phospholipids in the emulsified mixture decreased (Fig. 3a), and the amount of internal phospholipids agreed well with that of the unaltered $\mathrm{PC}$ after the phospholipase $\mathrm{A}_{2}$ treatment. Moreover, as no phosphorus signal of lamella PC was observed, the result suggests that most of the internal phospholipids were composed of PC. PC has been known to form (a)

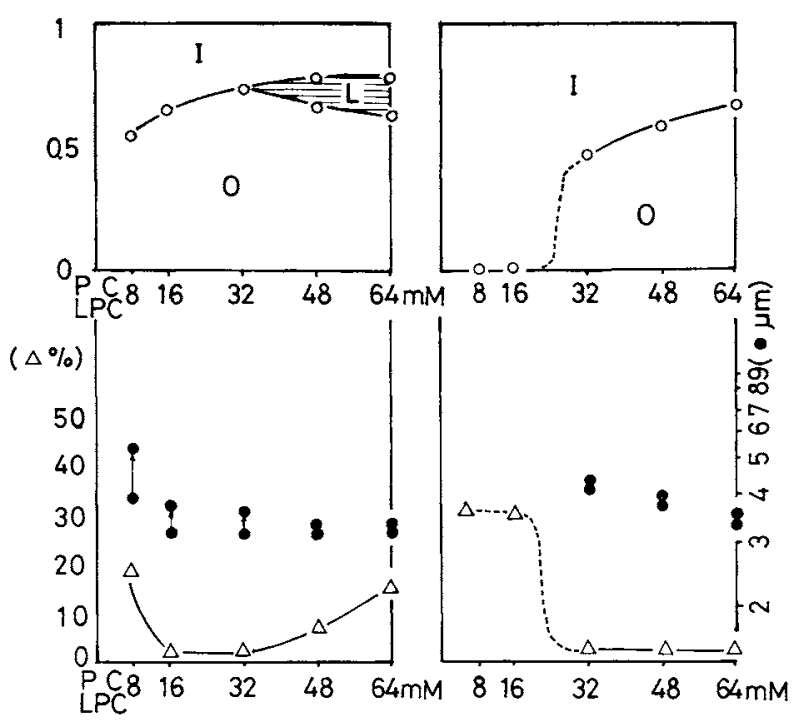

Fig. 3. Proportion of Phospholipids in the Internal (I) and External (O) Aqueous Phase and in the Multilamellar Vesicle (L).

The emulsions were composed of equimolar mixtures of PC and LPC of various concentrations, $N$-decane and an aqueous phase (a, deionized water; $b, 10 \mathrm{~mm} \mathrm{HCl}$ ). The molarity on the abscissa indicates the respective concentration of PC and LPC in the aqueous phase. The stability of the corresponding emulsions shown below was evaluated by the proportion of separated aqueous layer $(\Delta)$ and the change of mean diameter $(\boldsymbol{O})$ of the emulsion droplets. The dotted line represents a phase inversion. 
a water pool in non-polar solvents ${ }^{17)}$; therefore, $\mathrm{PC}$ can be presumed to participate in the formation of an internal aqueous phase in the emulsified mixture of PC and LPC.

Relationship between the phosphorus signals in the presence of $\mathrm{Pr}^{3+}$ and the emulsion stability

In the emulsion under deionized aqueous phase conditions, the ratio of the separated aqueous phase was lower than $5 \%$ when the concentration of phospholipids was in the range of $16 \mathrm{~mm}$ to $32 \mathrm{~mm}$ (Fig. 3). When the concentration of phospholipids was less than $8 \mathrm{~mm}$, the mean diameter of the oil particles increased markedly during standing. The ratio of the separated aqueous phase become higher and small amount of lamella could be observed as the concentration of phospholipids increased; however, the distribution of particle size was almost unchanged during $24 \mathrm{hr}$ standing after emulsification. In acidic conditions, the amount of internal phospholipids was large in comparison with the deionized conditions, and no lamella signal was observed even at a high concentration of phospholipids. The emulsion in the acidic condition showed a small change of particle size and low proportion of the separated aqueous layer. However, when the concentration of phospholipids was less than $16 \mathrm{~mm}$, a phase inversion occurred to separate the aqueous layer during standing. These results suggest that, in the emulsion containing concentrated phospholipids in deionized water, excess phospholipids in the continuous aqueous phase promoted aggregation of the oil particles. As the particle size of the emulsion, however, was scarcely increased, it can be presumed that the oil particles were prevented from merging with each other by the phospholipids in the continuous phase. In addition, oil particles in the diluted phospholipid conditions merged to increase their size, and the proportion of the separated aqueous layer became larger as the amount of phospholipids decreased. (a)

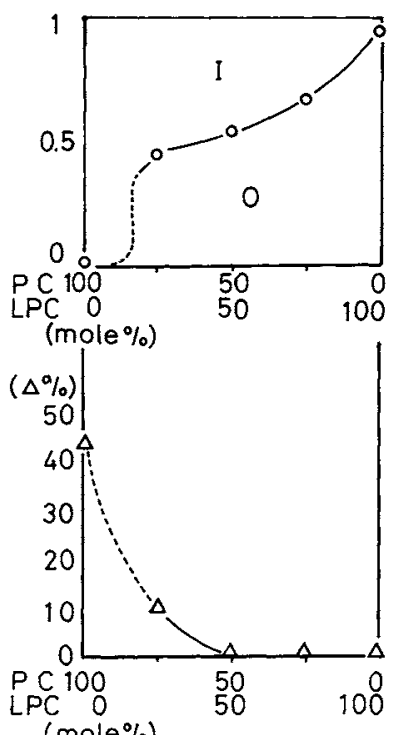

(b)
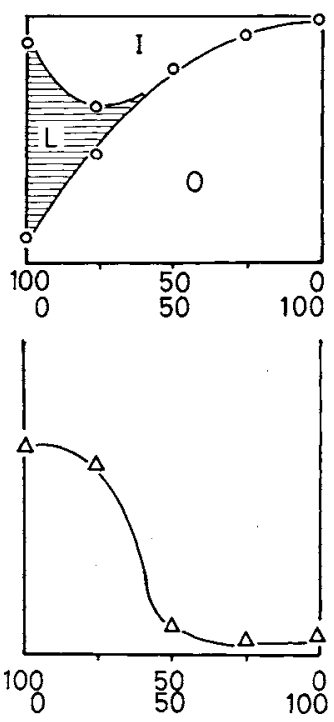

(c)
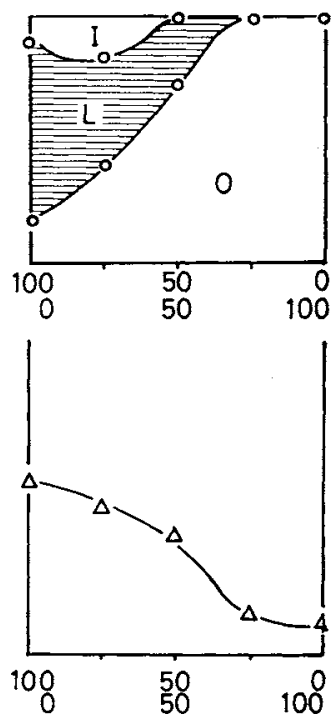

Fig. 4. Phospholipid Ratios of $1 / O / L$ (See Fig. 3) in the Emulsions Composed of $64 \mathrm{~mm}$ Phospholipids (PC and LPC in Various Compositions), $n$-Decane and an Aqueous Phase (a, $10 \mathrm{~mm} \mathrm{HCl} ; \mathrm{b}, 10 \mathrm{~mm} \mathrm{NaCl} ; \mathrm{c}, 10 \mathrm{~mm}$ $\mathrm{NaOH}$ ).

Below is shown the ratio of the separated aqueous layer of the corresponding emulsions. The dotted line represents a phase inversion. 
Emulsion stability with various $P C / L P C$ ratios

The amount of internal phospholipids and the emulsion stability change with the ratio of PC/LPC. Figure 4 shows the ratio of phospholipids on the internal and external interfaces and in the lamella state. In the aqueous phase of $10 \mathrm{~mm} \mathrm{HCl}$, the ratio of phospholipids inside increased corresponding to the amount of PC. No lamella signal was observed by ${ }^{31} \mathrm{P}$ NMR, and a phase inversion occurred when the $\mathrm{PC} / \mathrm{LPC}$ ratio was more than 3 . On the other hand, in the aqueous conditions of $10 \mathrm{~mm} \mathrm{NaCl}$ or $\mathrm{NaOH}$, the amount of the separated aqueous layer in the emulsion became markedly large with the existence of lamella signals. These results suggest that the oil particles became prone to aggregate in the presence of multilamellar PC. This was also confirmed by comparing the separation rates of the aqueous phase between the emulsions of LPC and those containing multilamellar PC which was added after emulsification. In the emulsion system composed of $n$-decane, deionized water and LPC in the range of $8 \mathrm{~mm}$ to $32 \mathrm{~mm}$, no aqueous phase was observed after $1 \mathrm{hr}$; however, in the emulsions containing $32 \mathrm{~mm} \mathrm{PC}, 2 \%$ to $15 \%$ of aqueous phase was separated.

\section{Conclusion}

The proportion of phospholipids located inside and outside the interfaces and in the lamella states was measured by ${ }^{31} \mathrm{P}-\mathrm{NMR}$ spectra. It varied on the aqueous phase conditions, and on the composition or concentration of the phospholipids. In a stable emulsion, most of the phospholipids were shown to be located on inside and outside the interfaces; however, with increasing of lamellar $\mathrm{PC}$, the ratio of $\mathrm{PC}$ inside decreased and separation of the aqueous layer was promoted.

PC and LPC are amphoteric emulsifiers, and hence their hydrophile-lipophile balance changes depending on the degree of dissociation of the phosphate and the quarternary ammonium residues. In an emulsion system composed of phospholipids, $n$-decane and water, a change of phospholipids distribution was clearly observed by ${ }^{31} \mathrm{P}-\mathrm{NMR}$ spectra, and was presumed to be correlated with the varied hydrophile-lipophile balance and the interfacial absorptive property of phospholipids.

\section{References}

1) J. E. Rothman and J. Lenard, Science, 195, 743 (1977).

2) J. G. Mandersloot, F. C. Reman, L. L. M. Van Deenen and J. D. Gier, Biochim. Biophys. Acta, 382, 22 (1975).

3) B. D. Kruyff, A. M. H. P. Van Den Beselaar and L. L. M. Van Deenen, Biochim. Biophys. Acta, $\mathbf{4 6 5}, 443$ (1977).

4) S. Carnie, J. N. Israelachvili and B. A. Pailthopre, Biochim. Biophys. Acta, 554, 340 (1974).

5) D. J. Murphy, FEBS Lett., 150, 19 (1982).

6) H. Hauser, Chem. Phys. Lipids, 43, 283 (1987).

7) S. Matsumoto, Y. Kita and D. Yonezawa, J. Colloid. Interface. Sci., 57, 353 (1976).

8) S. Matsumoto, M. Kohda and S. Murata, J. Colloid. Interface. Sci., 62, 149 (1977).

9) J. I. Howell, D. Fisher, A. H. Goodall, M. Verrinder and J. A. Lucy, Biochim. Biophys. Acta, 332, 1 (1973).

10) D. A. N. Morris, R. McNeil, F. J. Castellino and J. K. Thomas, Biochim. Biophys. Acta, 599, 380 (1980).

11) K. Chiba and M. Tada, Nippon Nōgeikagaku Kaishi, 62, 859 (1988).

12) K. Chiba and M. Tada, Agric. Biol. Chem., 53, 995 (1989).

13) E. A. Dennis and A. Pluckthun, in "Phosphorus-31 NMR. Principles and Applications," Academic Press, New York, 1984, Chapter 14.

14) I. C. Jones and G. R. A. Hunt, Biochim. Biophys. Acta, 820, 48 (1985)

15) C. M. Gupta, R. Radhakrishnan and H. G. Khorana, Proc. Natl. Acad. Sci. U.S.A., 74, 4315 (1977).

16) J. C. Wilschut, J. Regts and G. Scherphoe, FEBS Lett., 98, 18 I (1979).

17) V. V. Kumar and P. Raghunathan, Lipids, 21, 764 (1986). 